LETTERS

\section{Increased serum concentrations of tissue plasminogen activator correlate with an adverse clinical outcome in patients with bacterial meningitis}

Bacterial meningitis is the most common serious infection of the central nervous system. It is still characterised by high mortality and morbidity in adults. In this disease extensive perpetuated inflammation with leucocyte invasion into the central nervous system (CNS) results in breakdown of the blood-brain barrier and promotes neuronal damage.

Tissue type plasminogen activator (tPA) has been shown to have various biological effects that could have an impact on the pathophysiological changes observed in bacterial meningitis. In the CNS, endothelial cells, microglia, astrocytes, and neurones can produce the 70 kDa protein tPA, which normally does no cross the blood-brain barrier. ${ }^{2}$ Raised tPA levels in the cerebrospinal fluid (CSF) have previously been reported for certain CNS diseases such as multiple sclerosis, leukaemia, and encephalitis, ${ }^{3}$ and raised serum tPA levels for patients with sepsis

tPA converts plasminogen into plasmin, a rate limiting step in the proteolysis of fibrin, but also in the degradation of extracellula matrix, matrix metalloproteinase activation, and the processing of growth factors and cytokines. ${ }^{2}$ Further, tPA has been shown to increase neuronal cell death during excitotoxicity and cerebral ischaemia. ${ }^{2}$ Thus tPA may promote blood-brain barrier disruption, proinflammatory signalling, and neuronal damage, and so be involved in the pathophysiology of bacterial meningitis.

We studied the expression of tPA in the CSF and serum of 12 patients with bacterial meningitis (causative pathogens: Str pneumoniae (8); $S$ aureus (3); $H$ influenzae (1)) who had been admitted to our hospital (median age 63 years; range 29 to 78 ). Clinical outcome was measured according to the Glasgow outcome scale (GOS; 1 , death; 2 , persistent vegetative state; 3 , severe disability; 4 , moderate disability; 5, good recovery). Ten patients with

A

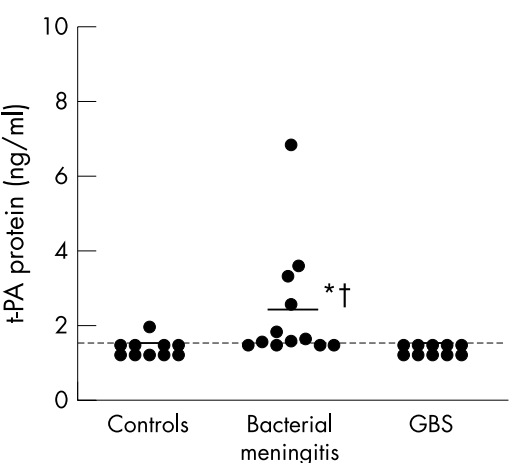

non-inflammatory neurological diseases (median age 37 years; range 23 to 81 ) and 10 patients with Guillain-Barré syndrome, an inflammatory demyelinating polyradiculoneuropathy in which blood-CSF barrier breakdown occurs without CSF pleocytosis, served as controls (median age 59 years; range 34 to 84 ).

A lumbar puncture was done and venous blood collected for diagnostic purposes after the patient's informed consent had been obtained. CSF and serum concentrations of tPA were measured by a specific enzyme linked immunosorbent assay (TintElize ${ }^{\circledR}$, Biopool International, Ventura, California, USA; detection limit $1.5 \mathrm{ng} / \mathrm{ml}$ ). Immunoreactive tPA concentrations are expressed as $\mathrm{ng} / \mathrm{ml}$ of biological fluid.

Blood and CSF variables for the three patient groups were compared using the Mann-Whitney U test with $\alpha$ adjustment; a corrected $p$ value of $<0.025$ was considered significant. Bivariate correlations between clinical variables and tPA concentrations were analysed according to Spearman $\rho$ (GOS) or Pearson (CSF leucocyte count, CSF/albumin ratio)

In all patients with bacterial meningitis, the CSF leucocyte count was markedly increased (median 1728 cells/ $\mu$ l; range 143 to 23296 ). The CSF to serum albumin ratio $(1000 \times \mathrm{CSF}$ albumin/serum albumin; normal $<7.4$ ), the index used to quantify blood-CSF barrier breakdown, was significantly increased in all patients with bacterial meningitis (median 60.3; range 156 to 1400 ) and, to a lesser extent, in nine of the 10 patients with Guillain-Barré syndrome (median 12.8; range 4.7 to 39.0 )

The tPA protein concentrations in the CSF and serum of patients with bacterial meningitis were increased compared with those of control patients and patients with GuillainBarré syndrome; in both of the latter groups, tPA concentrations in the CSF were not detectable in nine of 10 patients (fig 1). The serum concentrations of tPA (mean (SD)) in patients with bacterial meningitis were about ninefold higher than the CSF concentrations (22.5 (13.8) v 2.4 (1.6) ng/ml, p < 0.05). CSF and serum concentrations in individual patients were positively correlated $(r=0.733$ $\mathrm{p}<0.01)$. Remarkably, high serum tPA concentrations in bacterial meningitis correlated with both an increased CSF to serum albumin

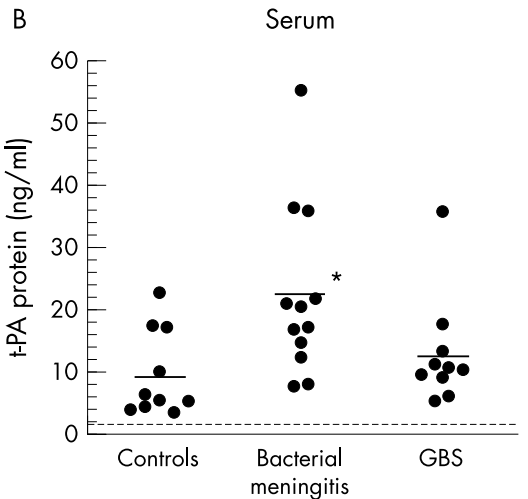

Figure 1 (A) Concentrations of tissue type plasminogen activator ( $\mathrm{PAA}$ ) in the cerebrospinal fluid (CSF) of control patients (controls, mean (SD): $1.54(0.15) \mathrm{ng} / \mathrm{ml}$ ), patients with bacterial meningitis (2.42 (1.59) ng/ml), and patients with Guillain-Barré syndrome (GBS, 1.50 (0.02) $\mathrm{ng} / \mathrm{ml}$ ). (B) Concentrations of PPA in the serum of controls (9.71 (6.92) $\mathrm{ng} / \mathrm{ml})$, patients with bacterial meningitis (22.51 (13.84) ng/ml), and patients with GBS (13.28 (8.74) ng/ml). ${ }^{*} p<0.025 v$ control patients; $\uparrow p<0.025 v$ patients with GBS. Dotted line: detection limit of the assay. ratio $(r=0.818, \mathrm{p}<0.01)$ and an unfavourable outcome according to the GOS $(r=-0.72, \mathrm{p}<0.01)$. The CSF to serum albumin ratio also showed a high correlation with CSF tPA concentrations $(r=0.942$, $\mathrm{p}<0.001)$. For patients with bacterial meningitis no correlations were found between serum tPA and CSF leucocyte count $(r=-0.319, \mathrm{p}=0.311)$, between CSF tPA and CSF leucocyte count $(r=-0.070, \mathrm{p}=0.828)$, or between CSF tPA and the clinical outcome $(\mathrm{r}=-0.201, \mathrm{p}=0.530)$

On the basis of these findings, we hypothesise that increased serum tPA contributes to breaching of the blood-brain/CSF barrier in bacterial meningitis. In turn, the breaching allows the serum tPA, which an intact bloodCSF barrier normally keeps separate from the CNS, to enter the CSF.

Our study shows for the first time that both CSF and serum tPA are increased in bacterial meningitis. Furthermore, upregulation of serum tPA correlated positively with breakdown of the blood-CSF barrier and an adverse clinical outcome of this disease. These findings are of particular importance in the light of earlier studies in rodent models, in which systemic infusion of tPA or plasmin resulted in blood-brain barrier disturbances in healthy control animals or in cerebral ischaemia. ${ }^{5}$ Disruption of the blood-brain/CSF barrier is an important pathophysiological alteration in bacterial meningitis, which contributes to CNS complications such as cerebral oedema and increased intracranial pressure. ${ }^{1}$ This may explain the additional correlation we found between high serum tPA levels and an adverse clinical outcome. A similar correlation was seen in patients with severe sepsis, a disease regularly associated with increased vascular permeability, in which serum tPA activity increased and was associated with mortality.

\section{Acknowledgement}

This study was supported by grants from the Förderprogramm Forschung und Lehre of the Ludwig-Maximilians University Munich (to FW) and from the Wilhelm Sander-Stiftung (to HWP). We thank Ms S Walter and B Angele for technical assistance and Ms J Benson for copy editing the manuscript

F Winkler, S Kastenbauer, U Koedel, H W Pfister

Department of Neurology, Klinikum Grosshadern, udwig-Maximilians University, Marchioninistr 15, D-81377 Munich, Germany

Competing interests: none declared.

Correspondence to: $\mathrm{Dr} \mathrm{H}-\mathrm{W}$ Pfister pfister@nefo.med.uni-muenchen.de

\section{References}

1 Pfister HW, Fontana A, Tauber MG, et al. Mechanisms of brain injury in bacterial meningitis: workshop summary. Clin Infect Dis 1994;19:463-79.

2 Strickland S. Tissue plasminogen activator in nervous system function and dysfunction. Thromb Haemost 2001;86:138-43.

3 Akenami FO, Siren V, Koskiniemi M, et al. Cerebrospinal fluid activity of tissue plasminogen activator in patients with neurological diseases. J Clin Pathol 1996;49:577-80.

4 Philippe J, Offner F, Declerck PJ, et al. Fibrinolysis and coagulation in patients with infectious disease and sepsis. Thromb infectious disease and sepsis.

5 Busch E, Kruger K, Fritze K, et al. Blood-brain barrier disturbances after rt-PA treatment of thromboembolic stroke in the rat Acta Neurochir Suppl 1997;70:206-8. 


\section{Amelioration of spinal myoclonus with levetiracetam}

Spinal myoclonus has been associated with various spinal cord insults, including mass lesions, ischaemia, infection, and as part of a paraneoplastic syndrome. 'It has been postulated that it occurs as a result of deficient inhibitory glycinergic transmission in the spinal cord and subsequent "release" of synchronous motor neurone oscillations within segments of the cord. Levetiracetam (UCB Pharma, Smyrna, Georgia, USA) is a new antiepileptic drug that has been shown recently to reduce the effect of glycinergic inhibitors. We describe three patients whose spinal myoclonus was markedly ameliorated by levetiracetam.

\section{Case reports}

Patient 1: spinal epidural compression

A 62 year old woman with known diffuse large cell lymphoma presented to her oncologist with progressive back pain accompanied by a band-like sensation around her waist. In the preceding four weeks, she had also been troubled by spontaneous involuntary abdominal contractions, and in the preceding two weeks these were accompanied by involuntary jerks of her legs. The patient could not suppress these spontaneous movements; moreover, as voluntary leg movements often precipitated them, she was unable to walk safely because of numerous falls. She denied any limb weakness and bladder or bowel incontinence.

On examination, she had a mild spastic paraparesis with $4+/ 5$ MRC grade power in a pyramidal pattern in the lower extremities (quadriceps, hamstrings, and tibialis anterior), 3+ knee and ankle jerks, and extensor plantar responses bilaterally. There were frequent resting myoclonic jerks of her lower extremities, involving both proximal and distal musculature, occurring at a rate of $150-250 / \mathrm{min}$. There were also occasional, infrequent resting myoclonic jerks affecting the trunk. The myoclonic jerks were exacerbated in amplitude during attempts to perform purposeful movements, suggesting the phenomenonology of action myoclonus. The abnormal movements, rather than weakness, made it impossible for her to stand or walk unassisted. Magnetic resonance imaging (MRI) of the spine revealed malignant infiltration of the lower thoracic vertebrae with evidence of cord compression at T11. An EEG was normal.

She was treated with a maximum tolerated dose of clonazepam ( $1 \mathrm{~g} /$ day) with minimal improvement. She was then started on levetiracetam $250 \mathrm{mg}$ twice daily, and within three days the resting and action myoclonus subsided markedly, such that she was able to walk with no assistance. On examination, the myoclonic jerk frequency in her lower extremities had decreased to $5-10 / \mathrm{min}$, and the jerk amplitude was markedly diminished.

Patient 2: zoster myelitis

An 85 year old woman presented with a three month history of involuntary trunk movements. The movements consisted of sudden extensor jerks of her back. They were spontaneous, occurring several times a day with no obvious provoking factors. Of note, two months before the onset of the movements, she had been diagnosed as having thoracic herpes zoster (at T8) and had subsequent postherpetic neuralgia. The back movements began as the pain was subsiding. The movements were not painful, but were distressing to the patient as they were socially embarrassing. She was unable to suppress the movements voluntarily. She had been seen by another neurologist who had treated the movements with sodium valproate. She unfortunately received no benefit from this despite a maximum tolerated dose of $2000 \mathrm{mg} /$ day. Past medical history was notable for cardiac arrhythmia and pacemaker placement.

On examination, she had brief, irregular, extensor movements of her thoracic spine, occurring every 10-30 seconds. An EEG was normal. MRI of the thoracic spine was precluded because of her pacemaker. The patient was given levetiracetam at a dose of $250 \mathrm{mg}$ twice a day. Within 24 hours of starting this treatment, the myoclonic movements completely ceased. Two months later, she began to have clusters of repetitive movements once to twice daily for periods of 20-60 minutes. Her dose of levetiracetam was increased to $500 \mathrm{mg}$ twice a day. The movements again ceased, but because of sedation and dizziness at this higher dose, the dosage was reduced to $375 \mathrm{mg} /$ day. At this well tolerated dose, she has been having brief clusters of myoclonic movements two or three times a week.

\section{Patient 3: transverse myelitis}

A 12 year old boy presented with a three month history of rhythmic spasms of his right thigh. One month before this symptom, he had had onset of bilateral leg weakness and paraesthesiae and was diagnosed as having acute transverse myelitis. The paraparesis largely resolved within two weeks of onset, but one month later he began having constant, rhythmic jerks of his right quadriceps and hamstrings. These jerks could not be suppressed voluntarily and made walking difficult. Cerebrospinal fluid analysis and an MRI of the spinal cord were normal. An EEG did not show any epileptiform activity. Sodium valproate $(1000 \mathrm{mg} / \mathrm{d})$, phenytoin $(300 \mathrm{mg} / \mathrm{d}$ ), and intravenous lorazepam (as often as $2 \mathrm{mg}$ every 4 hours) failed to relieve the constant myoclonus. A trial of botulinum toxin A injections into the right quadriceps did not ameliorate the movements.

On examination, he had constant, semirhythmical myoclonus of his right quadriceps at $120-150$ beats/min with his knee extended, and of his right hamstrings with his knee flexed. The myoclonus was not suppressed by patellar fixation, but did improve slightly with concentration on mental tasks. On power testing, there was $4+/ 5$ MRC grade power in the right quadriceps and right hamstrings. A repeat EEG was again unremarkable. He was started on levetiracetam at $250 \mathrm{mg}$ daily and the dose increased over a four week period to $1250 \mathrm{mg} / \mathrm{d}$. No clinical change was noted until the $1250 \mathrm{mg}$ dose was reached, at which point the myoclonus slowed and then completely stopped over a seven day period, allowing independent ambulation. Other than mild initial sedation, no side effects were experienced.

\section{Discussion}

Glycine is a major inhibitory neurotransmitter in the spinal cord, and it has been postulated that deficient inhibitory glycinergic transmission results in dysfunction of segmental spinal cord circuitry, and hence a myoclonic focus in the spinal cord. This postulate is based on studies of animal models of myoclonus ${ }^{2}$ and an in vitro model of spinal myoclonus. ${ }^{3}$ The latter study showed that blockade of glycine receptors in isolated spinal cord preparations from neonatal rats en- hanced a central pattern generator responsible for 5 to $15 \mathrm{~Hz}$ synchronous motor neurone oscillations. Interestingly, these oscillationsgenerated from as few as two isolated segments-were synchronised over at least six spinal cord segments, suggesting extensive excitatory commissural connections.

It is possible that the effectiveness of levetiracetam in our patients may be related to these glycinergic mechanisms. Levetiracetam has been shown to reverse inhibition of glycine and GABA gated currents induced by negative allosteric modulators, such as zinc and $\beta$-carbolines. ${ }^{4}$ It may therefore conceivably be of benefit in patients with spinal myoclonus by augmenting glycinergic transmission in the spinal cord and thus dampening down myoclonic foci.

In a recent open labelled trial of levetiracetam in eight patients with chronic myoclonus, three of five patients with cortical myoclonus experienced reduction in their myoclonus severity, as assessed by the unified myoclonus rating scale. ${ }^{5}$ The one patient in this study with spinal myoclonus showed no improvement with levetiracetam. However, the average duration of symptoms in these patients was 7.6 years, ranging from one to 17 years, in contrast to our three patients whose symptoms were one to three months in duration before levetiracetam treatment. It is therefore possible that the differential responsiveness to levetiracetam was because the aforementioned non-responder had a chronic fixed condition whereas our responders had subacute evolving spinal cord injuries.

In a recently published study, levetiracetam was used successfully to treat three patients with posthypoxic and postencephalitic myoclonus, two of whom had failed to respond to valproic acid and clonazepam. ${ }^{6}$ Add-on therapy with levetiracetam was shown to suppress disabling post-hypoxic cortical reflex myoclonus in a 16 year old boy. ${ }^{7}$ In another study, severe action myoclonus was suppressed by levetiracetam in three patients, of whom two had Unverricht-Lundborg disease and one had postanoxic myoclonus. ${ }^{8}$

Our cases, as well as the abovementioned reports of suppression of post-hypoxic and postencephalitic myoclonus with levetiracetam, suggest that this agent is promising for the treatment of both non-cortical and cortical myoclonus. These observations will need to be confirmed in additional patients. Furthermore, the proportion of responders needs to be determined in a larger group of patients, ideally in the setting of a randomised, double blind, placebo controlled trial.

S C Keswani, E H Kossoff, G L Krauss Department of Neurology, The Johns Hopkins University, 600 North Wolfe Street, Baltimore, Maryland 21287, USA

C Hagerty

Neurology Specialists, Columbia, Maryland, USA

Competing interests: GLK is a paid consultant to UCB Pharma. The terms of this arrangement are being managed by the Johns Hopkins University in accordance with its conflict of interest policies. The other authors declare no competing interests.

\section{Correspondence to: Dr G L Krauss;} gkrauss@jhmi.edu

\section{References}

1 Brown P, Thompson P, Rothwell J, et al. Axial myoclonus of propriospinal origin. Brain 1991;114:197-214.

2 Gundlach A. Disorder of the inhibitory glycine receptor: inherited myoclonus in Poll Hereford calves. FASEB J 1990;4:2761-6. 
3 Simon ES. Involvement of glycine and $G A B A_{A}$ receptors in the pathogenesis of spinal myoclonus. Neurology 1995;45: 1883-92.

4 Rigo JM, Nguyen L, Belachew S, et al. Levetiracetam: novel modulation of ionotropic inhibitory receptors. Epilepsia 2000;41/supp 7):35

5 Frucht SJ, Louis ED, Chuang C, et al. A pilot tolerability and efficacy study of levetiracetam in patients with chronic myoclonus. Neurology 2001:57:1112-14.

6 Krauss GL, Bergin A, Kramer RE, et al. Suppression of post-hypoxic and post-encephalitic myoclonus with levetiracetam. Neurology 2001;56:411-12.

7 Schaver $\mathbf{R}$, Singer $M$, Saltuari $L$, et al. Suppression of cortical myoclonus by levetiracetam. Mov Disord 2002;17:41 1-15.

8 Genton P, Gelisse P. Antimyoclonic effect of levetiracetam. Epileptic Disord 2000;2:209-12

\section{Hyperthyroidism with increased factor VIII procoagulant protein as a predisposing factor for cerebral venous thrombosis}

Cerebral venous thrombosis (CVT) is a rare disorder, with an incidence of approximately 4/1 000000 per year, occurring more frequently in women than in men (ratio of 1.29:1). ${ }^{1}$ CVT is a multifactorial condition known predisposing factors include venous stasis, hypercoagulability, vasculitis, systemic lupus erythematosus, and trauma. Mortality after CVT ranges from 5\% to $30 \%{ }^{1}$ The optimal treatment consists of anticoagulation for six months and should only be maintained beyond this time if known risk factors for CVT persist. Treatment should not be discontinued in case of an asymptomatic haemorrhagic transformation of the associated venous infarct. $^{2}$

In recent years, a few thyrotoxic patients with CVT have been reported. An association between hyperthyroidism and increase of FVIII has also been described, ${ }^{3}$ and recent data suggest an increased incidence of venous thrombosis in patients with hyperthyroidism and high FVIII levels. ${ }^{4}$ Here we report patient with increased FVIII levels and an autoimmune hyperthyroidism, who devel oped a CVT complicated by venous infarction.

\section{Case report}

A 39 year old woman was admitted to the emergency room after a brief episode of convulsions, preceded by a short period of perseveration, verbal aggressiveness, and disorientation. Four days before admission, she had developed a sudden, pulsatile left sided headache, which was unresponsive to paracetamol and ibuprofen. Personal and family medical histories were unremarkable. She had been taking oral contraceptive medication for several years and smoked two cigarettes a day. Neurological examination was normal, except for a temporary confusional state that lasted less than 24 hours. Electroencephalography demonstrated a slow arrhythmia in the left temporal region, without epileptic activity. Brain computed tomography revealed a left temporal hypodense lesion, with moderate contrast enhancement. Magnetic resonance imaging of the brain performed 24 hours later, showed a non-specific hyperintense lesion on the $\mathrm{Tl}$ weighted images. The magnetic resonance venography (fig l) revealed an extensive thrombosis of the left lateral sinus with involvement of the distal part of the jugular vein. The diagnosis of a temporal venous infarct was made. Treatmen with unfractionated heparin was started promptly and maintained for one week, followed by oral anticoagulation with an INR between 2 and 3. Oral contraceptive treatment was discontinued and the patient was advised to stop smoking. Extensive screening for coagulopathies including antiphospholipid syndrome, dysfibroginaemia, deficiencies in antithrombin, protein $\mathrm{C}$ and $\mathrm{S}$, hyperhomocysteinaemia, and activated protein $C$ resistance revealed no abnormalities. The G20210A prothrombin gene mutation was absent Autoimmune tests including ANF, ANCA complement and rheumatoid factors were negative. Further analysis revealed a state of hyperthyroidism with a TSH value below $0.015 \mathrm{mIU} / \mathrm{l}$ (normal: $0.27-4.2$ ), free triiodothyronin of $12.1 \mathrm{ng} / \mathrm{l}$ (normal: 9.3-18.0 $\mathrm{ng} / \mathrm{l}$ ), and an increased free thyroxin of 28.8 ng/l (normal: 9.3-18.0 ng/l). Anti-TSH receptor antibodies were found consistent with Graves-Basedow's disease. The patient was treated with thiamazole $(3 \times 10 \mathrm{mg} / \mathrm{day})$, followed by the administration of radioactive iodine ( $9 \mathrm{mCi})$. One month after discontinuation of oral contraceptives, thyroid tests remained increased. FVIII procoagulant protein showed a marked increase: 1680 IU/l (normal levels: 500-1500 IU/1) and remained slightly raised five weeks later. Meanwhile the patient developed a hypothyroidism, necessitating a substitution treatment with LT4. After a further six months both thyroid tests and FVIII levels normalised and anticoagulants were stopped.

\section{Discussion}

Increase of clotting FVIII occurs in several conditions such as strenuous exercise, fever, pregnancy, renal failure, adrenaline (epinephrine) infusion, prednisone treatment, and intravascular haemolysis. ${ }^{3}$ Hyperthyroidism, whatever its origin, also induces a significant increase in FVIII levels, with a comparatively short activated partial thromboplastin time while other clotting factors remain within normal limits ${ }^{3}$ Moreover, correction of thyroid function results in a normalisation of FVIII levels. In patients with recurrent hyperthyroidism, levels of FVIII are known to fluctuate with thyroid function. The physiopathological mechanism involved remains unclear. Excessive adrenergic activity occurring in hyperthyroid patients could have a direct effect on the production of FVIII. The fact that administration of propanolol inhibits the increase of FVIII in patients with hyperthyroidism supports this theory.

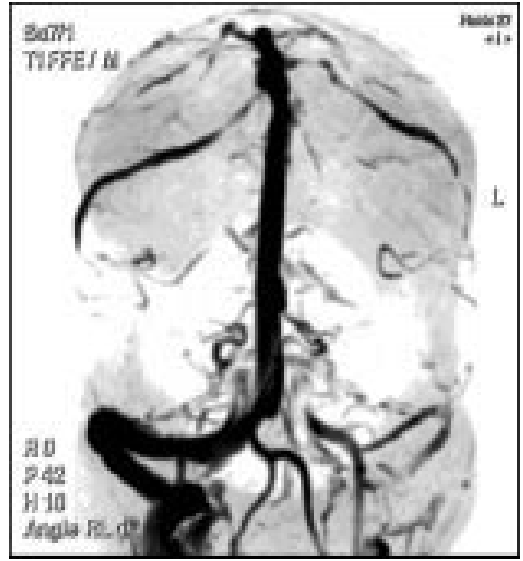

Figure 1 Magnetic resonance venography confirms complete occlusion of the left lateral sinus.
In 1995 a large study was performed on 301 case-control pairs, younger than 70 with a first episode of deep vein thrombosis. ${ }^{4}$ Patients with malignant disorders were excluded. The authors showed that high levels of FVIII contribute to the development of venous thrombosis in a dose dependent manner. In multivariate analysis FVIII concentrations above $1500 \mathrm{IU} / \mathrm{l}$ result in a 4.8 -fold higher risk of developing venous thrombosis. It was also shown that this is not an acute phase reaction, and that high levels of FVIII persist for months after the thrombotic event. Recently, it was calculated that the reported incidence of CVT and hyperthyroidism is significantly higher than expected by chance alone. ${ }^{5} \mathrm{~A}$ small number of case reports mention the concomitant occurrence of thyrotoxicosis and CVT. To our knowledge, this is the first reported case of CVT of the left lateral sinus associated with clinically silent hyperthyroidism and increased FVIII levels. Correction of thyroid function resulted in normalisation of FVIII levels. This report emphasises the need for thyroid evaluation in every patient with CVT and other venous thromboembolic events, even in the absence of clinical signs of hyperthyroidism. Every patient with hyperthyroidism, especially if immobilised, has a significantly higher risk of developing venous thromboembolism and should benefit from maximal preventive measures.

J Maes, A Michotte

Department of Neurology, Academisch Ziekenhuis, Vrije Universiteit Brussels, Laarbeeklaan 101, 1090 Brussels, Belgium

B Velkeniers

Department of Internal Medicine, Division of Endocrinology, Academisch Ziekenhuis, Vrije Universiteit Brussels

T Stadnik

Department of Radiology and Medical Imaging, Academisch Ziekenhuis, Vrije Universiteit Brussels

K Jochmans

Department of Clinical Biology, Division of Haematology, Academisch Ziekenhuis, Vrije Universiteit Brussels

Competing interests: none declared

Correspondence to: Dr J Maes; maesjen@belgacom.net

\section{References}

1 Ameri A, Bousser M. Cerebral venous

thrombosis. Neurol Clin 1992;10:87-111.

2 Einhaüpl K, Villringer A, Meister W, et al. Heparin treatment in sinus venous thrombosis. Lancet 1991;338:597-600.

3 Simone J, Abildgaard C, Schulman I. Blood coagulation in thyroid dysfunction. $N$ Engl J Med 1965;273:1057-61

4 Koster T, Blann A, Briët $E$, et al. Role of clotting factor VIII in effect of von Willebrand factor on occurrence of deep-vein thrombosis. Lancet 1995;345:152-5.

5 Verberne $\mathbf{H}$, Fliers E, Prummel M, et al. Thyrotoxicosis as a predisposing factor for cerebral venous thrombosis. Thyroid 2000; 10:607-61

\section{Coma with focal neurological} signs caused by Datura stramonium intoxication in a young man

Intoxication with Datura stramonium, which contains a variety of tropine alkaloids, produces atropine-like effects. The seeds of $D$ stramonium (semen stramonii) in particular contain hyoscyamine, scopolamine, and atropine. Symptoms include agitation, disorientation, hallucination, flushed skin, dilatation of 
pupils, urine retention, seizures, and respiratory depression. ${ }^{1} D$ stramonium is voluntarily used for its hallucinogenic properties. ${ }^{2}$ Its anticholinergic compounds are likely to produce delirium and stupor but rarely cause deep coma. ${ }^{3}$

A common diagnostic error is to mistake coma resulting from brainstem infarction, supratentorial mass lesions, metabolic disorders, or hypoxia for coma resulting from poisoning. The initial distinction of these conditions may be difficult. ${ }^{4}$ We report an unusual case of $D$ stramonium intoxication in a patient who initially presented with deep coma, focal neurological signs, and decorticate posture.

A 30 year old male patient was admitted to an emergency unit for acute loss of consciousness. The accompanying person reported that the patient had had a few beers and then suddenly fell on his back. He was unconscious and awoke for a few seconds but shortly afterward lost consciousness again and remained in a stiff position and unconscious until admission.

The first neurological examination was performed one and a half hours after the sudden onset of symptoms. There was no evidence of trauma. Vital signs, such as cardiopulmonary function, body temperature, and blood oxygenation, were normal. Initial laboratory testing for electrolyte disorders, renal or hepatic failure, and hypoglycaemia or hyperglycaemia found no major pathology. Blood alcohol concentration was $1.1 \%$.

Neurologically, he presented in a decorticate posture. The upper limbs were in a paratonic flexor position with increase of flexion tonus to noxious stimuli, which was more pronounced on the right side. The lower extremities did not respond to noxious stimuli and remained in an extensor position, which was also slightly more pronounced on the right side. Both the upper and the lower extremities greatly resisted passive motion. The patient had no verbal responses. The eyes could not be opened with verbal or painful stimuli. Both pupils were completely dilated and not reactive to light. The eyeballs were divergent. Corneal responses were bilaterally absent.

The horizontal oculocephalic response, however, was intact, while the vertical response was minimal. Swallowing reflex was minimal but also intact. Respiratory patterns were regular. Deep tendon reflexes could not be evaluated because of the massive increased muscle tone. Plantar response was extensor, bilaterally, more prominent on the right side. Tachycardia and retention of urine were also present. Initially the patient scored four on the Glasgow coma scale.

Magnetic resonance imaging of the brain was performed to detect brainstem infarction or supratentorial mass lesions. There were no pathological findings. Common metabolic disorders such as hypoglycaemia or hyperglycaemia, hepatic or renal failure, electrolyte disorders, disorders of systemic acid-base balance, and hyperthyroidism were excluded by laboratory examinations. Urine samples for benzodiazepines and morphines were negative. Analysis of cerebrospinal fluid to exclude subarachnoidal haemorrhage or infectious disease showed normal cell count, protein concentration, and cytology. Possible status epilepticus was also considered. However, administration of $10 \mathrm{mg}$ diazepam had no effect.

The next neurological examination was performed three hours later. Vital signs were stable. The upper limbs were still in a flexor position and the lower limbs were still extensor; however, the increased muscle tone began to decrease and was less resistant to passive motion. He withdrew abnormally from painful stimuli. Plantar response was extensor on the right side. The pupils were still dilated and not reactive to light but both corneal reflexes were intact. No verbal responses could be obtained. He now scored six on the Glasgow coma scale. Seven hours later he was sitting in his bed in a state of confusion. Over the next hours, the patient's neurological signs subsided gradually.

Finally, we were informed about the intake of $D$ stramonium seeds. Analysis of blood samples found increased concentrations of alkaloids. Treatment during the clinical course was supportive with cardiopulmonary monitoring. Thirty six hours after admission the patient was discharged in good clinical condition, without neurological deficits except amnesia regarding the acute toxic episode.

Coma from exogenous poisons or drugs is a common diagnostic problem, not only because of the variety of clinical symptoms but also because of incomplete medical histories and misguided efforts by families and friends to conceal facts. Even if a particular toxic agent is suspected, results of a chemical analysis may arrive too late. Therefore, an accurate and immediate diagnosis depends mostly on the clinical findings.

Our patient presented with coma in a decorticate posture. Initially a severe multifocal brainstem infarction or supratentorial mass lesions were suggested. However, the discrepancies of deep coma, absent brainstem reflexes such as corneal reflexes and nonreactive dilated pupils, and, on the other hand, the intact oculocephalic and swallowing reflex, and especially the regular respiratory patterns made the findings inconclusive and a toxicological cause probable. Moreover, vital signs were stable and magnetic resonance imaging of the brain, cerebrospinal fluid, and laboratory examinations showed no major pathological findings.
$D$ stramonium is misused for its hallucinogenic effects. It can be obtained as a herb, as a powder, and as seeds. The typical anticholinergic effects of D stramonium are well known. Coma with focal neurological signs and decorticate posture is an unusual presentation of $D$ stramonium intoxication. However, the presence of coma in our patient was linked to the atropine effect, described as the central anticholinergic syndrome, which has been reported in the literature.

Physostigmine, which may reverse anticholinergic toxicity, was not administered because it can produce severe complications such as seizures and cardiac arrhythmia. ${ }^{14}$ Moreover, the patient's neurological symptoms subsided gradually.

Regarding this uncommon clinical presentation, the pharmacological interaction between ethanol and D stramonium must also be taken into account. However, as far as we are aware, no clinical or pharmacological interactions between ethanol and $D$ stramonium in humans have been described in the literature.

$D$ stramonium intoxication with the clinical picture of coma, decorticate posture, and focal neurological signs is an important clinical observation, which must be taken into account in other comatose states.

S Oberndorfer, W Grisold

Department Neurology and LBI for Neurooncology, Kaiser Franz Josef Hospital, Kundratstrasse 3, 1100

Vienna, Austria

G Hinterholzer, M Rosner

Intensive Care Unit, Kaiser Franz Josef Hospital, Kundratstrasse 3, 1100 Vienna, Austria

Competing interests: none declared

Correspondence to: $\mathrm{Dr}$ S Oberndorfer; stefan.oberndorfer@kfj.magwien.gv.at

\section{References}

1 Rocco VG. Poisoning. In: Young GB, Ropper $\mathrm{AH}$, Bolton CF, eds. Coma and impaired consciousness. New York: McGraw-Hill, 1998;16:460-2.

2 Castanon Lopez L, Martinez Badas JP, Lapena Lopez De Armentia S, et al. Datura stramonium poisoning. An Esp Pediatr 2000;53:53-5.

3 Giubelli D, Conti L, Bott A, et al. Atropine poisoning. Importance of the clinical diagnosis. Minerva Anestesiol 1998;64:567-73.

4 Plum F, Posner JB, eds. The diagnosis of stupor and coma. Contemporary neurological series. Philadelphia: FA Davis,

1982;4: 177-83.

5 Pardo Moreno J, Castro del Rio M, Martin Estefania $C$, et al. Secondary coma from atropine poisoning. An Med Interna 2001;18:164-5 